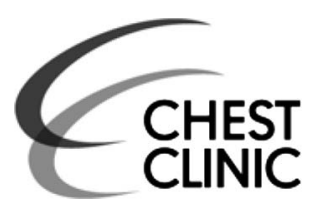

\title{
Developmental determinants in non-communicable chronic diseases and ageing
}

\author{
J Bousquet, ${ }^{1,2,3,4}$ J M Anto, ${ }^{4,5,6,7,8}$ K Berkouk, ${ }^{9}$ P Gergen, ${ }^{10}$ J Pinto Antunes, ${ }^{11}$ \\ P Augé, ${ }^{3,12}$ T Camuzat, ${ }^{3,13}$ J Bringer, $^{3,14} \mathrm{~J} \mathrm{Mercier,}^{3,15,16} \mathrm{~N}$ Best, $^{3,17} \mathrm{R}$ Bourret, $^{3,18}$ \\ M Akdis, ${ }^{4,19} \mathrm{~S} \mathrm{H}$ Arshad, ${ }^{20}$ A Bedbrook, $^{3}$ C Berr, ${ }^{3,21}$ A Bush, ${ }^{22} \mathrm{G}$ Cavalli, ${ }^{3,23}$ \\ M A Charles, ${ }^{24} \mathrm{~F}$ Clavel-Chapelon, ${ }^{25} \mathrm{M}$ Gillman, ${ }^{26,27} \mathrm{D}$ R Gold, ${ }^{28} \mathrm{M}$ Goldberg, ${ }^{29}$ \\ J W Holloway, ${ }^{30} \mathrm{P}$ lozzo, ${ }^{31} \mathrm{~S}$ Jacquemin, 3,32 C Jeandel, ${ }^{3,33} \mathrm{~F}$ Kauffmann, 4,34 \\ T Keil, 4,35,36 G H Koppelman, 4,37 S Krauss-Etschmann, ${ }^{38}$ D Kuh, ${ }^{39} \mathrm{~S}$ Lehmann, ${ }^{3,40}$ \\ K C Lodrup Carlsen, ${ }^{4,41}$ D Maier, ${ }^{4,42}$ M Méchali, ${ }^{23}$ E Melén, ${ }^{4,43}$ J P Moatti, ${ }^{44}$ \\ I Momas, ${ }^{4,45,46}$ P Nérin, 3,47 D S Postma, ${ }^{4,48}$ K Ritchie, $^{3,49} \mathrm{~J}$ M Robine, 3,50,51,52 \\ B Samolinski, ${ }^{53}$ V Siroux, ${ }^{4,54,55,56}$ P E Slagboom, ${ }^{57,58}$ H A Smit, ${ }^{4,59}$ J Sunyer, $^{4,5,6,7,8}$ \\ R Valenta, ${ }^{4,60} \mathrm{P}$ Van de Perre, ${ }^{61} \mathrm{~J} \mathrm{M}$ Verdier, ${ }^{3,62,63,64} \mathrm{M}$ Vrijheid, ${ }^{5,7,8}$ \\ M Wickman, ${ }^{4,43} \mathrm{P}$ Yiallouros, ${ }^{65} \mathrm{M}$ Zins $^{66}$
}

\section{- Additional material is} published online only. To view please visit the journal online (http://dx.doi.org/10.1136/ thoraxjnl-2014-206304).

TC representing $\mathrm{Mr} C$ Bourquin, President of the Région Languedoc Roussillon (C Bourquin, deceased author)

For numbered affiliations see end of article.

\section{Correspondence to} Professor Jean Bousquet, 273 av d'Occitanie, Montpellier 34090, France; fabienne.portejoie@gmail.com

Received 14 October 2014 Revised 22 October 2014 Accepted 24 October 2014 Published Online First 23 January 2015

\section{ABSTRACT}

Prenatal and peri-natal events play a fundamental role in health, development of diseases and ageing

(Developmental Origins of Health and Disease (DOHaD)). Research on the determinants of active and healthy ageing is a priority to: (i) inform strategies for reducing societal and individual costs of an ageing population and (ii) develop effective novel prevention strategies. It is important to compare the trajectories of respiratory diseases with those of other chronic diseases.

Prenatal and peri-natal events play a fundamental role in health, development of diseases and ageing (Developmental Origins of Health and Disease $(\mathrm{DOHaD}))$. Research on the determinants of active and healthy ageing (AHA) is a priority to: (i) inform strategies for reducing societal and individual costs of an ageing population and (ii) develop effective novel prevention strategies.

The European Union (EU) leads a global effort to understand the early determinants of ageing. The Polish Presidency of the EU Council (2011) targeted chronic respiratory diseases in children to promote AHA. ${ }^{1}$ The developmental determinants of chronic diseases in ageing were reinforced during the Cyprus Presidency of the EU Council (2012). Several projects of the EU Sixth and Seventh Framework Programme for Research and Technological Development (FP6 and FP7) were funded to understand the mechanisms of prenatal and early life events on the development of chronic diseases. One of the action plans of the European Innovation Partnership on AHA is devoted to integrated care for chronic diseases, and chronic respiratory diseases have been selected as the model for chronic diseases.

As part of this research initiative, a meeting in Montpellier on 2 and 3 December 2013 attempted to better understand the early life events that may impact ageing in health and disease, with a particular focus on respiratory diseases. The results of the FP6 and FP7 projects on population-based cohorts in adults and old age were reviewed to propose novel research, policies and value creation. The full report is in the online supplement and on the MACVIA-LR website (macvia.crlanguedocroussillon.fr).

\section{IMPORTANCE OF LIFE COURSE MODELS TO UNDERSTAND HEALTHY AGEING AND DISEASE}

Biological ageing is the progressive deterioration of function that occurs in the postmaturity phase and can be assessed at the individual, physiological systems and cellular levels. ${ }^{2}$ Cross cohort comparisons of British birth cohorts show that social and biological factors from early life onwards can affect ageing, in particular respiratory function and disease.

In order to detect health-promoting factors, the early life events of diseases such as COPD need to be understood (European COST action BM1201: Developmental Origins of Chronic Respiratory Disease $\left.^{3}\right)$. Susceptibility to COPD is associated with markers of foetal growth and early childhood disadvantage. Lung function at birth is determined by in utero processes.

Future research needs to develop a life course model of ageing that integrates the rather separate research on specific diseases or clinical conditions, functional ageing and well-being and should include socio-economic characteristics.

\section{BIRTH COHORTS FOCUSING ON CHRONIC RESPIRATORY DISEASES IN THE GENERAL POPULATION}

Asthma and allergic diseases begin early in life. Birth cohort studies are relevant to investigate environmental and lifestyle determinants of asthma and IgE-associated diseases or the absence of such diseases. Over 30 community-based birth cohorts 
focusing on asthma and allergies have started in Europe and many have been pooled. ${ }^{4}$ The Network of Excellence GA ${ }^{2}$ LEN (FP6, contract No FOOD-CT-2004-506378) has initiated birth cohort harmonisation. Two concerted actions, CHICOS (FP7 No. 241604) and ENRIECO (FP7 grant agreement No 226285), have built a network of more than 70 birth cohorts across Europe. Mechanisms of the Development of ALLergy (MeDALL, FP7 No. 261357) attempts to generate novel knowledge on the mechanisms of allergy onset and proposes early diagnosis, prevention and targets for therapy based on a new standardised follow-up of birth cohorts in Europe.

Numerous cohort studies have shown that early decrements in spirometry persist into late middle age, underscoring the crucial importance of early life influences. Optimal lung development depends on normal airway function at birth as well as normal growth during childhood up to the plateau of spirometric function around 25 years of age. After this, lung function declines as a normal event.

The geographical and temporal diversities of birth cohorts in MeDALL provide an excellent opportunity to study the effects of living conditions in different places since these are likely to be major determinants in the causation of the wave of chronic diseases currently observed.

An NIAID, NHLBI and MeDALL joint workshop (September $2012)^{5}$ identified over 60 birth cohorts focusing on asthma and allergy worldwide in order to (i) document the knowledge obtained, (ii) identify the knowledge gaps and inconsistencies and (iii) develop strategies for moving forward. Following the workshop in 2012, an online database containing information about existing cohorts was created to facilitate collaboration (AsthmaBirthCohorts.niaid.nih.gov).

Some population-based birth cohorts (PIAMA, BAMSE, ECA, MAS) have information on chronic diseases and their risk factors and can assess chronic diseases and AHA across the life cycle.

\section{NUTRITION, OBESITY AND DIABETES ACROSS THE LIFE COURSE}

Chronic diseases often start early in life and their processes expand over the life course. DORIAN (Developmental ORigins of healthy and unhealthy AgeiNg: The Role of Maternal Obesity, FP7) aims at linking studies of early developmental processes with those of ageing from a life course perspective.

\section{A better knowledge on the developmental origin of obesity and type-2 diabetes may lead to preventive measures in order to reduce the global epidemic of these two diseases.}

Nutrition is vital for health and disease prevention. The Supplementation with Antioxidant Vitamins and Minerals (SU. VI.MAX) study was a controlled trial (12 741 persons) followed up for 8 years (1994-2002) to test the efficacy of dietary manipulations in lowering the incidence of chronic diseases and cancer. No supplementation was administered to participants in SU.VI.MAX 2. Food intake and nutritional factors in infancy and childhood have also been studied in several of the ongoing European birth cohorts, which allows for unique longitudinal association analyses on various chronic diseases.

Nutrition is vital to understand the development of chronic diseases and the promotion of healthy ageing.

\section{ADULT COHORTS IN CHRONIC DISEASES}

The French prospective cohort (E3N), the EPIC-France cohort, is composed of women who were under a health insurance plan, the Mutuelle Générale de l'Education Nationale, for schoolteachers and coworkers. In 1990, about 100000 women (40-65 years) were recruited. Epidemiological research in nutrition, hormones and chronic diseases (asthma, diabetes or osteoporosis) studied the risk factors for developing these diseases.

Two large European epidemiological cohorts, recruited in the early 1990s and followed up for 20 years, have been carried out in respiratory health: (i) the European Community Respiratory Health Survey (ECRHS; http://www.ecrhs.org/), including 18668 individuals aged 20-44 years at baseline and (ii) the Epidemiological study of the Genetics and Environment of Asthma, bronchial hyperresponsiveness and atopy (EGEA; http://egeanet.vjf.inserm.fr), a French case-control and family study of adults and children at baseline, including 2120 individuals aged 7-70 years at baseline. Combined analyses between these cohorts have been performed.

COPACETIC, a cohort of the lung cancer screening trial with over 2200 individuals with lung function and CT scans, identified genes associated with lung function decline in interaction with smoking, CT scan-based emphysema and coronary calcifications.

LifeLines (The Netherlands) is a three-generation cohort of 165000 individuals followed annually by questionnaire and, every 5 years, by extensive functional screening of the five prime research areas: (1) metabolic/hormonal, (2) heart/vessel/kidney, (3) lung/respiratory/allergy, (4) psychiatric and (5) musculoskeletal.

Adult cohorts include participants that have been followed up for a long period of time, and may be used to link the development of factor profiles of chronic diseases from early life.

\section{OLD AGE COHORTS}

Neuropsychiatric disorders of old-age (eg, depression, Alzheimer's disease) may be determined by earlier risk exposure. The DEVELAGE consortium (FP7) aims to characterise shared molecular pathways between the early developmental processes in the brain and brain ageing.

This example underlines the limits of old age cohorts in the study of chronic neuropsychiatric disorder in the elderly.

Integrated research on DEvelopmental determinants of Ageing and Longevity (IDEAL; http://www.ideal-ageing.eu, FP7) examines the role of epigenetic regulation and transmission to next generations. A unique human cohort and animal studies are studied to discover novel longevity pathways and the links between development and ageing.

The Three-City Study (3C Study) is a population-based longitudinal study of the relation between vascular diseases and dementia in persons aged 65 years and older. A total of 9294 participants of both sexes were recruited from three French cities.

CONSTANCES, a large general-purpose epidemiological population-based cohort, aims to provide public health information. The cohort is a representative sample of 200000 French adults, aged 18-69 years at inception.

Cohorts in middle and old age adults can be intertwined with earlier cohorts to understand the mechanisms of AHA and diseases in ageing. 
Recommendations for future research are reported in the online supplement and the MACVIA-LR website on (i) integration of omics in epidemiological studies across the life cycle, (ii) performance of longitudinal studies assessing chronic diseases and (iii) value of European birth cohorts to study chronic diseases and AHA. Cohorts with an initial focus on chronic respiratory diseases could be expanded to understand the trajectories of chronic diseases and AHA.

Cohorts across the life cycle are essential to understand AHA. Lifelong birth cohorts give an overview of the problem and are complemented by cohorts carried out at different ages. Birth cohorts initially focusing on chronic respiratory diseases offer highly valuable additive information to understand chronic diseases and AHA.

\section{Author affiliations}

${ }^{1}$ University Hospital, Montpellier, France

${ }^{2}$ Inserm U 1168, Paris, France

${ }^{3}$ Contre les Maladies Chroniques pour un Vieillissement Actif en Languedoc Roussillon, Site de Référence de I'EIP on AHA, Montpellier, France

${ }^{4}$ MeDALL, Mechanisms of the Development of Allergy, FP7, Amsterdam, Groningen, the Netherlands

${ }^{5}$ Centre for Research in Environmental Epidemiology (CREAL), Barcelona, Spain

${ }^{6}$ IMIM (Hospital del Mar Medical Research Institute), Barcelona, Spain

${ }^{7}$ Universitat Pompeu Fabra (UPF), Barcelona, Spain

${ }^{8}$ CIBER Epidemiología y Salud Pública (CIBERESP), Barcelona, Spain

${ }^{9}$ Deputy Head of Unit for Medical Research and the Challenge of Ageing, DG Research \& Innovation, European Commission, Brussels, Belgium

${ }^{10}$ National Institute of Allergy and Infectious Diseases, Bethesda, Maryland, USA

${ }^{11}$ European Commission, Directorate General for Health and Consumers, Brussels, Belgium

${ }^{12}$ University Montpellier 1, France

${ }^{13}$ Région Languedoc Roussillon, France

${ }^{14}$ Montpellier Medical School, France

${ }^{15}$ Department of Physiology, Montpellier University Hospital, France

${ }^{16}$ University Montpellier 1, France

${ }^{17}$ Nimes University Hospital, France

${ }^{18}$ Montpellier University Hospital, France

${ }^{19}$ Swiss Institute of Allergy and Asthma Research (SIAF), Davos and University of Zurich, Switzerland

${ }^{20}$ David Hide Asthma and Allergy Research Centre, Isle of Wight, UK

${ }^{21}$ Inserm, Research Unit U1061, University Montpellier I, Montpellier, France

${ }^{22}$ Department of Paediatric Respiratory Medicine, Royal Brompton Hospital and National Heart and Lung Institute, Imperial College, London, UK

${ }^{23}$ Institute of Human Genetics, CNRS, Montpellier, France

${ }^{24}$ Equipe 10 UMR Inserm-Université Paris-Sud (Centre de recherche en Epidémiologie et Santé des Populations, CESP), Villejuif, France

${ }^{25}$ Nutrition, Hormones and Women's Health Team, INSERM UMR-S 1018, Paris-

South University, Villejuif, France

${ }^{26}$ Obesity Prevention Program, Department of Population Medicine, Harvard Medical School/Harvard Pilgrim Health Care Institute, Boston, Massachusetts, USA

${ }^{27}$ Department of Nutrition, Harvard School of Public Health, Boston, Massachusetts, USA

${ }^{28}$ Channing Division of Network Medicine, Department of Medicine, Brigham and Women's Hospital, Harvard Medical School, and Harvard School of Public Health, UK

${ }^{29}$ Population-Based Epidemiological Cohorts, INSERM-UVSQ UMS 011, Villejuif, France

${ }^{30}$ Human Development \& Health, Faculty of Medicine, University of Southampton, Southampton, UK

${ }^{31}$ Institute of Clinical Physiology, National Research Council (CNR), Pisa, Italy

${ }^{32}$ Horiba, Montpellier, France

${ }^{33}$ Department of Geriatrics, University Hospital, Montpellier, France

${ }^{34}$ CESP-Team of Respiratory and Environmental Epidemiology INSERM UMR-

S1018, University Paris-Sud, Villejuif, France

${ }^{35}$ Institute of Social Medicine, Epidemiology and Health Economics, CharitéUniversitätsmedizin, Berlin, Germany

${ }^{36}$ Institute for Clinical Epidemiology and Biometry, Julius Maximilian University of Wuerzburg, Germany

${ }^{37}$ Department of Pediatric Pulmonology and Pediatric Allergology, University of Groningen, University Medical Center Groningen, Beatrix Children's Hospital, Groningen Research Institute for Asthma and COPD, Groningen, The Netherlands
${ }^{38}$ Comprehensive Pneumology Center, Ludwig Maximilians University and Helmholtz Zentrum Muenchen, Member of the German Research Center for Lung Research, Großhadern, Germany

${ }^{39} \mathrm{MRC}$ Unit for Lifelong Health and Ageing at UCL, London, UK

${ }^{40}$ Institut de Médecine Régénératrice et de Biothérapie (I.M.R.B.), University Hospital, INSERM U1040, Montpellier, France

${ }^{41}$ Department of Paediatrics, University of Oslo and Oslo University Hospital, Oslo, Norway

${ }^{42}$ Biomax Informatics AG, Planegg, Germany

${ }^{43}$ Institute of Environmental Medicine, Karolinska Institutet and Sachs' Children's Hospital, Stockholm, Sweden

${ }^{44}$ Aix-Marseille University (AMU), Research Unit 912 AMU/INSERM/IRD Social and Economic Sciences Applied to Health (SESSTIM), France

${ }^{45}$ Department of Public health and biostatistics, Descartes University, Paris, France

${ }^{46}$ Municipal Department of social action, childhood, and health, Paris, France

${ }^{47}$ SATT AxLR, Montpellier, France

${ }^{48}$ Department of Pulmonology, University Medical Center Groningen, University of Groningen, GRIAC Research Institute, Groningen, The Netherlands

${ }^{49}$ Inserm U1061 Neuropsychiatry, Montpellier and Faculty of Medicine, Imperial

College London, London, UK

${ }^{50}$ Inserm Research Unit 988, Paris, France

${ }^{51}$ Inserm Research Unit 710, Montpellier, France

${ }^{52}$ Ecole Pratique des Hautes Etudes (EPHE), Paris, France

${ }^{53}$ Department of Prevention of Environmental Hazards and Allergology, Medical University of Warsaw, Warsaw, Poland

${ }^{54}$ Team of Environmental Epidemiology applied to Reproduction and Respiratory Health, University Grenoble Alpes, IAB, Grenoble, France

${ }^{55}$ Team of Environmental Epidemiology applied to Reproduction and Respiratory Health, INSERM, IAB, Grenoble, France

${ }^{56}$ Team of Environmental Epidemiology applied to Reproduction and Respiratory Health, CHU de Grenoble, IAB, Grenoble, France

${ }^{57}$ Section of Molecular Epidemiology, Leiden University Medical Center, Leiden, the Netherlands

${ }^{58}$ Consortium for Healthy Aging, Leiden University Medical Center, Leiden, the Netherlands

${ }^{59}$ Julius Center of Health Sciences and Primary Care, University Medical Center Utrecht, University of Utrecht, Utrecht, the Netherlands

${ }^{60}$ Division of Immunopathology, Department of Pathophysiology and Allergy Research, Center for Pathophysiology, Infectiology and Immunology, Medical University of Vienna, Vienna, Austria

${ }^{61}$ University Hospital and INSERM U 1058, Montpellier, France

${ }^{62} \mathrm{EPHE}$, Section des Sciences de la Vie et de la Terre, Paris, France

${ }^{63}$ UMR S 710, University Montpellier 2, Montpellier, Paris, France

${ }^{64}$ Institut Transdisciplinaire d'Etudes du Vieillissement, Montpellier, France

${ }^{65}$ Cyprus International Institute for Environmental \& Public Health in Association with Harvard School of Public Health, Cyprus University of Technology, Limassol, Cyprus

${ }^{66}$ Director of Population-Based Epidemiological Cohorts, INSERM-UVSQ UMS 011, Villejuif, France

Correction notice This article has been corrected since it was published Online First. The statement 'PG and JPA did not participate in the discussion' on the title page has been removed. It has now been corrected and included in the 'Contributors' statement.

Contributors All authors participated in the meeting. All authors contributed to the discussion, except for KB and JPA.

Funding The meeting was supported by an unrestricted educational grant from the Région Languedoc Roussillon awarded by Mr. C Bourquin (President).

Competing interests None declared.

Provenance and peer review Not commissioned; internally peer reviewed.

\section{REFERENCES}

1 Samolinski B, Fronczak A, Wlodarczyk A, et al. Council of the European Union conclusions on chronic respiratory diseases in children. Lancet 2012;379:e45-6.

2 Kuh D, Cooper R, Hardy R, et al. A life course approach to healthy ageing. Oxford: Oxford University Press, 2014.

3 Krauss-Etschmann S, Bush A, Bellusci S, et al. Of flies, mice and men: a systematic approach to understanding the early life origins of chronic lung disease. Thorax 2013;68:380-4.

4 Bousquet J, Anto J, Sunyer J, et al.; MeDALL Study Group; CHICOS Study Group; ENRIECO Study Group; GA²LEN Study Group. Pooling birth cohorts in allergy and asthma: European Union-funded initiatives - a MeDALL, CHICOS, ENRIECO, and GA(2)LEN joint paper. Int Arch Allergy Immunol 2013;161:1-10.

5 Bousquet J, Gern JE, Martinez FD, et al. Birth cohorts in asthma and allergic diseases: Report of a NIAID/NHLBI/MeDALL joint workshop. J Allergy Clin Immunol 2014; 133:1535-46. 